\title{
Species-specific identification and differentiation of Arcobacter, Helicobacter and Campylobacter by full-spectral matrix-associated laser desorption/ ionization time of flight mass spectrometry analysis
}

\author{
Correspondence \\ Claudia Hess \\ claudia.hess@vetmeduni.ac.at
}

Received 9 October 2009

Accepted 1 December 2009

\author{
Merima Alispahic, ${ }^{1}$ Karin Hummel, ${ }^{2}$ Delfina Jandreski-Cvetkovic, ${ }^{1}$ \\ Katharina Nöbauer, ${ }^{2}$ Ebrahim Razzazi-Fazeli, ${ }^{2}$ Michael Hess $^{1}$ \\ and Claudia Hess ${ }^{1}$
}
${ }^{1}$ Clinic for Avian, Reptile and Fish Medicine, Department for Farm Animals and Veterinary Public Health, University of Veterinary Medicine, Vienna, Austria
${ }^{2}$ VetOMICS Core Facility for Research/Proteomics and Metabolomics, University of Veterinary Medicine, Vienna, Austria

\begin{abstract}
Rapid and reliable identification of Arcobacter and Helicobacter species, and their distinction from phenotypically similar Campylobacter species, has become increasingly important, since many of them are now recognized as human and/or animal pathogens. Matrix-associated laser desorption/ ionization-time of flight (MALDI-TOF) MS has been shown to be a rapid and sensitive method for characterization of micro-organisms. In this study, we therefore established a reference database of selected Arcobacter, Helicobacter and Campylobacter species for MALDI-TOF MS identification. Besides the species with significance as food-borne pathogens - Arcobacter butzleri, Helicobacter pullorum, Campylobacter jejuni and Campylobacter coli - several other members of these genera were included in the reference library to determine the species specificity of the designed MALDI Biotyper reference database library. Strains that made up the reference database library were grown on Columbia agar, and yielded reproducible and unique mass spectra profiles, which were compared with the Bruker Biotyper database, version 2. The database was used to identify 144 clinical isolates using whole spectral profiles. Furthermore, reproducibility of MALDI-TOF MS results was evaluated with respect to age and/or storage of bacteria and different growth media. It was found that correct identification could be obtained even if the bacteria were stored at room temperature or at $4{ }^{\circ} \mathrm{C}$ up to 9 days before being tested. In addition, bacteria were correctly identified when grown on Campylosel agar; however, they were not when grown on modified charcoal cefoperazone deoxycholate agar. These results indicate that MALDI-TOF MS fingerprinting is a fast and reliable method for the identification of Arcobacter and Helicobacter species, and their distinction from phenotypically similar Campylobacter species, with applications in clinical diagnostics.
\end{abstract}

\section{INTRODUCTION}

Identification of species belonging to the Arcobacter, Helicobacter and Campylobacter genera has become increasingly important, since many of them are recognized as human and/or animal pathogens. Arcobacter butzleri was found to be the fourth most frequently isolated Campylobacter-like organism in human clinical samples,

Abbreviations: ATCC, American Type Culture Collection; MALDI, matrixassociated laser desorption/ionization; NCTC, National Collection of Type Cultures; TOF, time of flight.

A table of identification data is available as supplementary material with the online version of this paper. before Campylobacter lari, but after Campylobacter jejuni, Campylobacter coli and Campylobacter fetus, in Belgium and in France (Prouzet-Mauleon et al., 2006; Vandenberg et al., 2004). On several occasions, Helicobacter pullorum has been isolated from poultry (Zanoni et al., 2007; Ceelen et al., 2007; Atabay et al., 1998; Neubauer \& Hess, 2006b). A number of research groups have associated $H$. pullorum with gastroenteritis, diarrhoea, and liver and gall bladder disease in human patients (Young et al., 2000; Stanley et al., 1994; Castera et al., 2006; Rocha et al., 2005). For $H$. pullorum there is a lack of phenotypic identification methods, and as a result, this bacterium is commonly misidentified as thermophilic Campylobacter (Atabay et al., 
1998; Kuijper et al., 2003). C. jejuni is the leading cause of bacterial gastroenteritis in developed countries (EFSA, 2005). In humans the majority (97\%) of food-borne diseases can be attributed to animals farmed for meat, especially poultry (Wilson et al., 2008).

Various molecular DNA-based methods for the identification of Arcobacter, Helicobacter and Campylobacter species have been developed. These methods typically require the use of several species-specific PCR primers, hybridization probes or multiple restriction enzymes, and are usually not designed to differentiate all known species simultaneously (Bohr et al., 2002; Jauk et al., 2003; Wilson et al., 2008; Neubauer \& Hess, 2006a). Bacterial identification by matrix-associated laser desorption/ionization-time of flight (MALDI-TOF) MS is based on generating complex fingerprints of biomarker molecules by measuring the exact mass/charge ratio of peptides and proteins (Claydon et al., 1996; Suh \& Limbach, 2004). A number of species from the Campylobacter genus (Mandrell et al., 2005; Kolinska et al., 2008) have been characterized by MALDI-TOF MS. In addition, Helicobacter pylori and Helicobacter mustelae were analysed by MALDI-TOF MS (Winkler et al., 1999), but not $H$. pullorum and Helicobacter pametensis. So far, no study applying MALDI-TOF MS based on the same sample preparation and technology, and using a whole spectral profile to differentiate potentially confounding Arcobacter and Helicobacter species together with phenotypically similar Campylobacter species has been reported.

In this study, we established a reference database of selected Arcobacter, Helicobacter and Campylobacter. The second objective was to use the reference database to identify 144 clinical isolates and compare the results to molecular methods. The third objective was to evaluate the reproducibility using different growth media and age of bacteria that are relevant in a diagnostic lab.

\section{METHODS}

Bacterial strains. For establishing the database library, reference strains were obtained from the National Collection of Type Cultures (NCTC) and from the American Type Culture Collection (ATCC). Preliminary work was done first on C. jejuni NCTC 12744, A. butzleri NCTC 12481 and H. pullorum ATCC 51801 to optimize the method. For standardizing the culture method all bacteria were grown on Columbia (COS) agar containing $5 \%$ sheep blood (bioMérieux), at $42{ }^{\circ} \mathrm{C}$ for $48 \mathrm{~h}$ under microaerobic conditions (GENbox microaer; bioMérieux). In addition, bacteria were grown on Campylosel (CAM) agar (bioMérieux) and on modified CCD (charcoal cefoperazone deoxycholate) agar [blood-free agar base with $32 \mathrm{mg}$ cefoperazone $1^{-1}$ and $10 \mathrm{mg}$ amphotericin $\mathrm{ml}^{-1}$ (Oxoid)] for reproducibility testing. Escherichia coli strain DH5 $\alpha$ (Invitrogen) was grown on COS agar at $37{ }^{\circ} \mathrm{C}$ for $24 \mathrm{~h}$.

Reference strains used in this study to generate the database library are listed in Table 1, which includes their origin, species and other information. The reference library was then used to identify 144 clinical isolates that were obtained from humans, the environment and from different farm animals, but mostly poultry (Table 2).

PCR-RFLP analyses. To identify the clinical isolates by molecular methods, the protocol of Jauk et al. (2003) was followed. Briefly, the isolates were examined by PCR-RFLP. PCR based on the 16S rRNA gene of the genera Arcobacter, Helicobacter and Campylobacter amplified a $1216 \mathrm{bp}$ fragment. The amplicons were digested with the restriction enzymes RsaI and EcoRV. Additional differentiation was obtained using PCR assay based on the hippuricase-encoding gene (Marshall et al., 1999).

Sample preparation for MALDI-TOF MS analysis. A single colony was removed from the agar plates using an inoculating loop and the material was placed in a vial containing $300 \mu$ MilliQ purified water to suspend the bacteria. To inactivate the bacteria, $900 \mu \mathrm{l}$ absolute ethanol was added to the vial. After centrifugation for $2 \mathrm{~min}$ at $20000 \mathrm{~g}$ the supernatant was removed. Afterwards, a second centrifugation step was done to remove the ethanol completely. For cell-wall disruption, $50 \mu \mathrm{l}$ formic acid (70\%) was added to the pellet and thoroughly mixed. Subsequently, $50 \mu$ l acetonitrile were added for protein extraction. After a centrifugation for $3 \mathrm{~min}$ at $20000 \mathrm{~g}$, $1 \mu$ supernatant containing the bacterial extract was transferred to a

Table 1. Reference strains used to establish the reference database for MALDI-TOF MS based species identification

\begin{tabular}{|c|c|c|c|c|}
\hline Designation & Genus & Species & Subspecies & Origin \\
\hline NCTC 12145 & Campylobacter & jejuni & jejuni & Human \\
\hline NCTC 12744 & Campylobacter & jejuni & jejuni & Contaminated milk \\
\hline ATCC 700819 & Campylobacter & jejuni & jejuni & Human faeces \\
\hline NCTC 12143 & Campylobacter & coli & & No information given \\
\hline NCTC 12144 & Campylobacter & lari & & Child with mild diarrhoea \\
\hline NCTC 11458 & Campylobacter & lari & & Child with mild diarrhoea \\
\hline ATCC 35217 & Campylobacter & hyointestinalis & & Swine with proliferative enteritis \\
\hline NCTC 10842 & Campylobacter & fetus & fetus & Brain of sheep fetus \\
\hline NCTC 12481 & Arcobacter & butzleri & & Human faeces \\
\hline ATCC 49616 & Arcobacter & butzleri & & Human faeces \\
\hline ATCC 49942 & Arcobacter & butzleri & & No information given \\
\hline ATCC 49615 & Arcobacter & cryaerophilus & & Human blood \\
\hline ATCC 51400 & Arcobacter & skirrowii & & Cow (abomasitis) \\
\hline ATCC 51801 & Helicobacter & pullorum & & Asymptomatic broiler chicken \\
\hline ATCC 51802 & Helicobacter & pullorum & & Human faeces \\
\hline ATCC 51478 & Helicobacter & pametensis & & Tern \\
\hline DH5 $\alpha$ & Escherichia & coli & & \\
\hline
\end{tabular}


Table 2. Species identification results for the clinical isolates using MALDI-TOF MS in comparison to PCR-RFLP

\begin{tabular}{|c|c|c|c|c|c|c|}
\hline \multirow{2}{*}{$\begin{array}{l}\text { Isolation and } \\
\text { identification }\end{array}$} & \multicolumn{6}{|c|}{ No. of isolates } \\
\hline & A. butzleri & H. pullorum & C. coli & C. jejuni & C. hyointestinalis & C. fetus \\
\hline Human & 3 & 8 & - & - & - & - \\
\hline Broiler chicken & - & 1 & 19 & 62 & - & - \\
\hline Bovine & - & - & 1 & 2 & 2 & - \\
\hline Cat & - & - & - & - & - & 1 \\
\hline Duck & - & - & - & 1 & - & - \\
\hline Environment & - & - & - & 3 & - & - \\
\hline \multicolumn{7}{|l|}{ Method } \\
\hline MALDI-TOF MS* & $3 / 3$ & $9 / 9$ & $57 / 57$ & $72 / 72$ & $2 / 2$ & $1 / 1$ \\
\hline
\end{tabular}

${ }^{*}$ MALDI-TOF MS represents an identification at species level $\log$ (score) value of $\geqslant 2.0$.

$\dagger$ PCR-RFLP analyses were carried out according to the protocol of Jauk et al. (2003).

sample position of a ground/polished steel MALDI target plate and allowed to dry at room temperature. Each sample was spotted six times onto the MALDI target plate to test technical replication. Then, the sample was overlaid with $2 \mu \mathrm{l}$ matrix ( $\alpha$-cyano-4-hydroxycinnamic acid in $50 \%$ acetonitrile $/ 2.5 \%$ trifluoroacetic acid) and dried again. All steps were performed at room temperature.

MALDI-TOF MS parameters. Mass spectra were collected using Ultraflex II MALDI-TOF/TOF mass spectrometer (Bruker) in linear mode, i.e. using a mass range of 2000 to $20000 \mathrm{Da}$ (parameter setting: IS1 $20.0 \mathrm{kV}$, IS2 $18.7 \mathrm{kV}$, lens $6.25 \mathrm{kV}$, detector gain $1634 \mathrm{~V}$ ). Five hundred single spectra $(10 \times 50$ laser shots $)$ were summarized with a $50 \mathrm{~Hz}$ nitrogen laser for each sample. The instrument was externally calibrated with E. coli strain DH5 $\alpha$ ribosomal proteins, e.g. RL36 $4364.3 \mathrm{~m} / \mathrm{z}$, RS32 $5095.8 \mathrm{~m} / \mathrm{z}$, RS34 $5380.4 \mathrm{~m} / \mathrm{z}$, RS33meth. 6254.4 $\mathrm{m} / \mathrm{z}$, RL29 $7273.5 \mathrm{~m} / \mathrm{z}$ and RS19 $10299.1 \mathrm{~m} / \mathrm{z}$.

Data visualization and analysis. Each individual spectrum was scrutinized by eye using the flexAnalysis software 3.0 (Bruker Daltonik) and atypical spectra were excluded from further analysis (e.g. flat line spectra, spectra containing high matrix background signal). A reference database library was established for MALDI-TOF MS-based species identification following the manufacturer's recommendations for Ultraflex measurement and the MALDI Biotyper 1.1 software package (Bruker Daltonik). In brief, for each database entry, at least 30 individually measured mass spectra fingerprints were imported into the MALDI Biotyper 1.1 software. Eight independent measurements (i.e. bacteria were grown at eight different times over the course of 3 months and subsequently measured) were obtained at six different spots each. After smoothing, baseline correction and peak-picking, the resulting peak lists (up to 70 peak masses) were used by the program to calculate and to store a main spectrum containing the information about mean peak masses, mean peak intensities and peak frequency.

Species identification of clinical isolates by MALDI-TOF MS. The MALDI-TOF MS reference database was used to identify and differentiate 144 clinical isolates. For correct identification of species, a generated peak list was matched against the established reference library using the integrated pattern-matching algorithm of MALDI Biotyper 1.1 software. Briefly, the software calculates $\log$ (score) values, that is, a $\log ($ score $)$ between 1.7 and 2.0 represents genus identification. A $\log$ (score) value of $\geqslant 2.0$ represents an identification at species level. Anything less than a $1.7 \log$ (score) was rated as not identifiable by the software.

MALDI-TOF MS reproducibility test. To test the reproducibility of MALDI-TOF MS-based species identification, 15 field strains were selected randomly and the reproducibility of their spectra under different conditions was tested. At first, bacteria were grown on COS agar for $48 \mathrm{~h}$ at $42{ }^{\circ} \mathrm{C}$. Subsequently they were stored for 2, 4, 6 and 9 days at room temperature $\left(20{ }^{\circ} \mathrm{C}\right)$, and at $4{ }^{\circ} \mathrm{C}$, to see if the age and/or the storage conditions of the bacteria have an influence on the results. Secondly, these selected strains were grown for $48 \mathrm{~h}$ at $42{ }^{\circ} \mathrm{C}$ on CAM and mCCD agars, which are regularly used to isolate such bacteria.

\section{RESULTS AND DISCUSSION}

Detection and characterization of infectious micro-organisms in a reasonably fast and reliable manner from biological and environmental samples has a high priority. The genera Arcobacter, Helicobacter and Campylobacter belong to the rRNA superfamily VI. The differentiation between members of these three genera is challenging, particularly when employing biochemical tests as the sole criterion due to the inconsistency of the phenotypic profiles observed among strains (On, 1996). To overcome the problems related to classical phenotypic species identification methods, this study evaluated the capability of MALDI-TOF MS to differentiate and identify these species. For comparison, PCR-RFLP, a classical genotypic method, was chosen to characterize clinical strains.

The MALDI-TOF MS reference database was established with 16 well-characterized culture collection strains from different sources representing 10 different species of Arcobacter, Helicobacter and Campylobacter. These species yielded reproducible and unique mass spectral profiles, 
which were compared with Bruker Biotyper database, version 2. However, the Bruker Biotyper database version 2 did not contain spectra from $H$. pametensis, as a result this bacterium did not match with any other spectra in the database. Therefore, the $H$. pametensis mass spectral profile is unique. All reference strains used represented the majority of clinically relevant species from the three genera. In addition, E. coli $\mathrm{DH} 5 \alpha$ spectra were included in the reference database library.

The most common Arcobacter species were analysed, namely A. butzleri, Arcobacter skirrowii and Arcobacter cryaerophilus (Fig. 1). Interestingly, mass signal patterns of A. cryaerophilus and A. skirrowii as well as A. butzleri and A. skirrowii shared a number of common mass peaks that were $100 \%$ frequent (in 135 spectra). Mass signal patterns of A. cryaerophilus were completely different to A. butzleri. However, the spectra of this A. cryaerophilus were found to have a number of small peak-shifts compared to the $A$. cryaerophilus type-strain in the Bruker Biotyper database, version 2. As a result, our reference $A$. cryaerophilus strain mass spectra profile did not reliably match the Bruker $A$. cryaerophilus type strain. This confirms the need to have a number of different strains of the same species coming from different sources in the reference database for correct identification.

Similarly, the two Helicobacter species, $H$. pullorum and $H$. pametensis (Fig. 2), had very different mass signal patterns and could be easily discriminated. Finally, five species from the genus Campylobacter widely found as human and/or animal pathogens were analysed, C. jejuni, C. coli, C. lari,
Campylobacter hyointestinalis and C. fetus. Representative fingerprint mass spectra clearly demonstrate that all five species produced unique molecular profiles and could be easily differentiated (Fig. 3). Within the species fingerprints, it was observed that for thermophilic Campylobacter (i.e. C. jejuni, C. coli and C. lari) the mass signal patterns were very different to the non-thermophilic Campylobacter (i.e. C. fetus and C. hyointestinalis). Therefore, the 10 species could be easily discriminated by means of a direct comparison of the whole mass spectra fingerprints.

Different mass spectrometric methods for analysing whole bacteria cells for intact proteins, including the identification of one or few specific protein biomarker ions, have been reported. Mandrell et al. (2005) focused on Campylobacter 'species-identifying' biomarker ions (SIBS) using MALDI-TOF MS and reported that there were SIBS types associated with the source. In addition, biomarker ions in the 9 to $14 \mathrm{kDa}$ range were reported to be diagnostic for Campylobacter species. However, one of the biomarkers [7035 Da see Fig. 3 (indicated by an asterisk)] published by Mandrell et al. (2005) was found to be $100 \%$ frequent (in 211 spectra) for thermophilic Campylobacter but not observed in non-thermophilic Campylobacter in this study. This biomarker was extracted and identified to be a 50 S ribosomal L29 protein by Fagerquist et al. (2006). In addition, specific C. jejuni biomarker $10276 \mathrm{Da}$ and specific C. coli biomarkers 10032 and 12855 Da proposed by Mandrell et al. (2005) were not frequently detected in this study. Therefore, species identification using one or few biomarker ions characteristic for a given species may

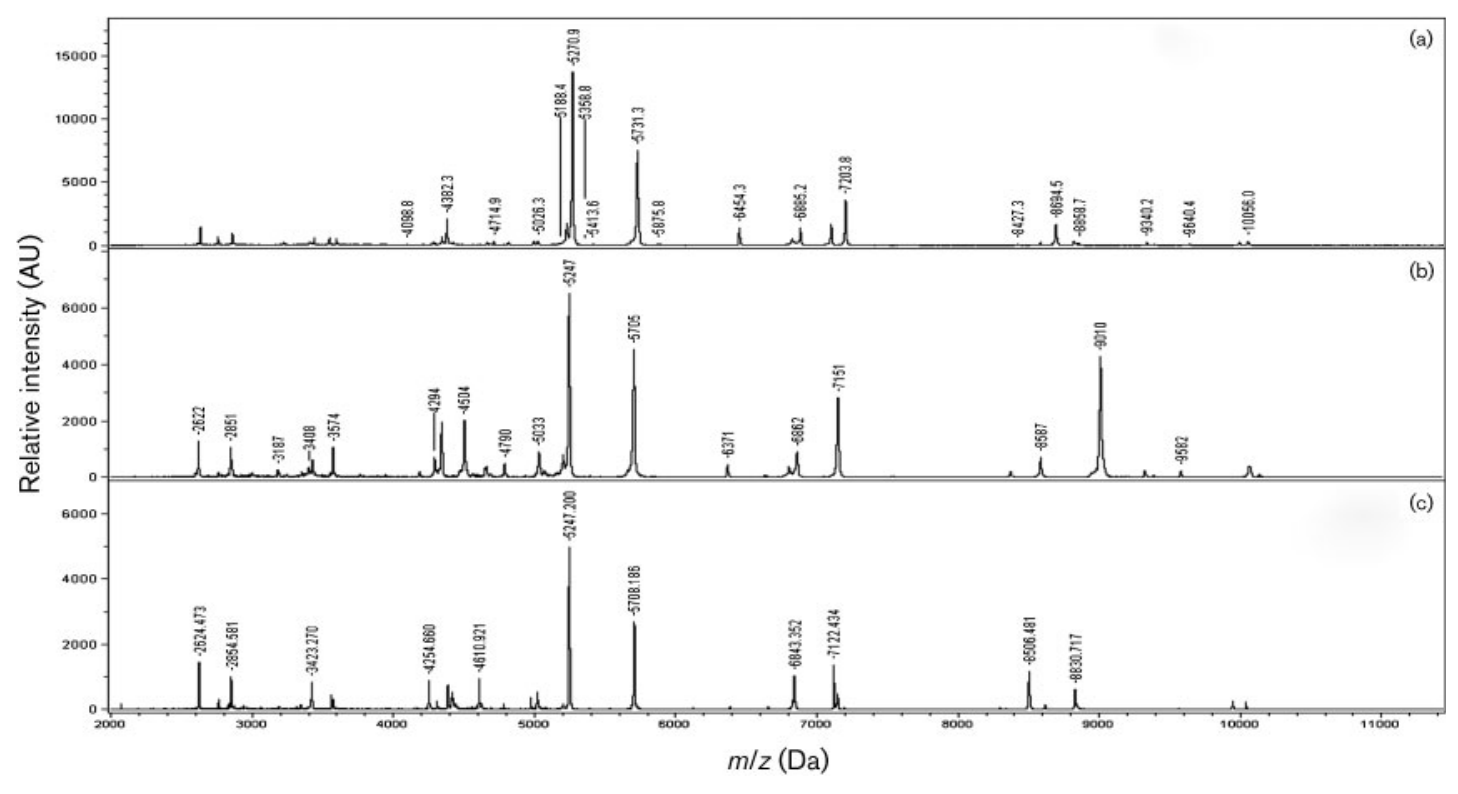

Fig. 1. MALDI-TOF MS profiles obtained from the analysis of $A$. butzleri (a), A. skirrowii (b) and A. cryaerophilus (c). The relative intensities of the ions [in arbitrary units $(\mathrm{AU})$ ] are shown on the $y$-axis, and the masses (in $\mathrm{Da}$ ) of the ions are shown on the $x$-axis. The $\mathrm{m} / \mathrm{z}$ value stands for mass to charge ratio. For a single positive charge, this value corresponds to the molecular mass of the protein. 


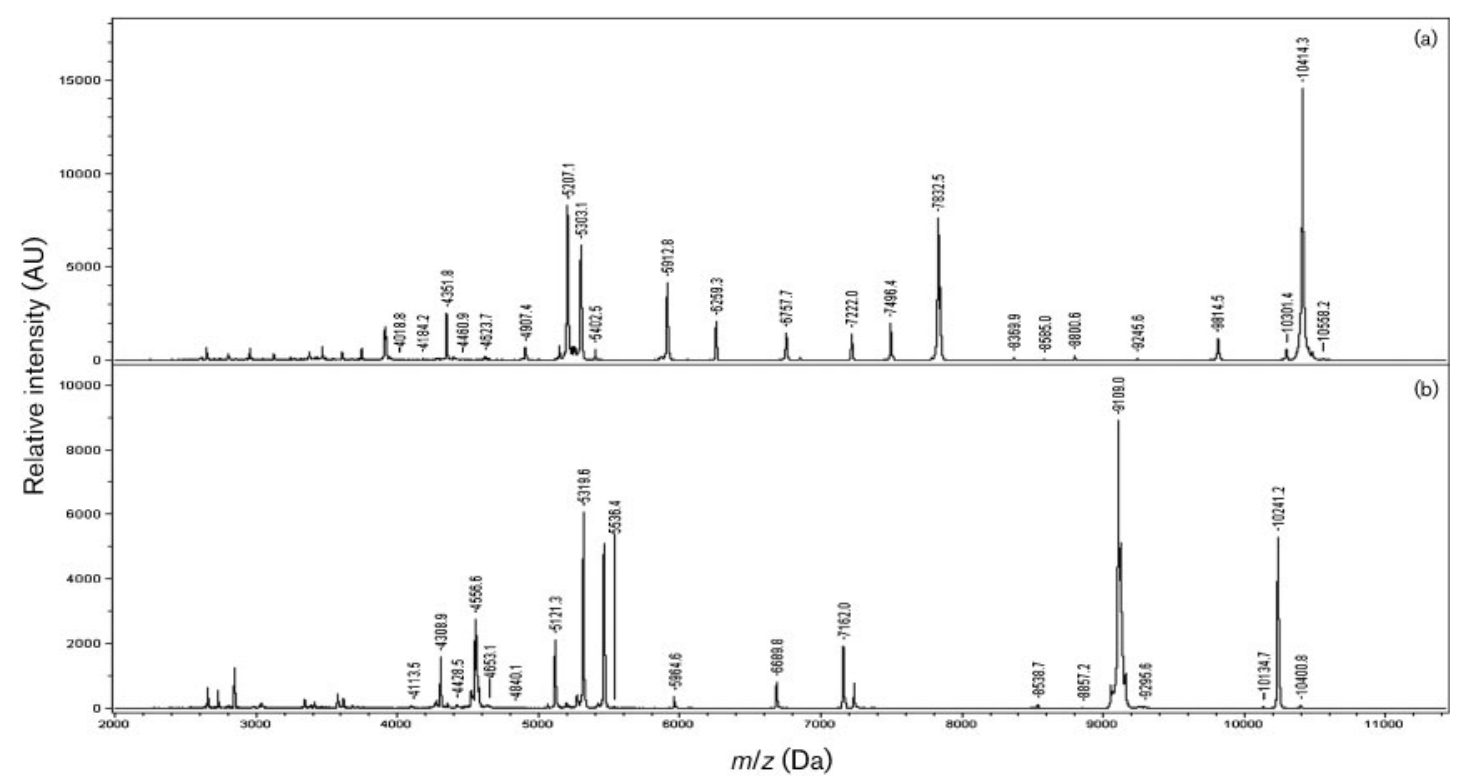

Fig. 2. MALDI-TOF MS profiles obtained from the analysis of $H$. pullorum (a) and $H$. pametensis (b). The relative intensities of the ions [in arbitrary units (AU)] are shown on the $y$-axis, and the masses (in Da) of the ions are shown on the $x$-axis. The $m / z$ value stands for mass to charge ratio. For a single positive charge, this value corresponds to the molecular mass of the protein.

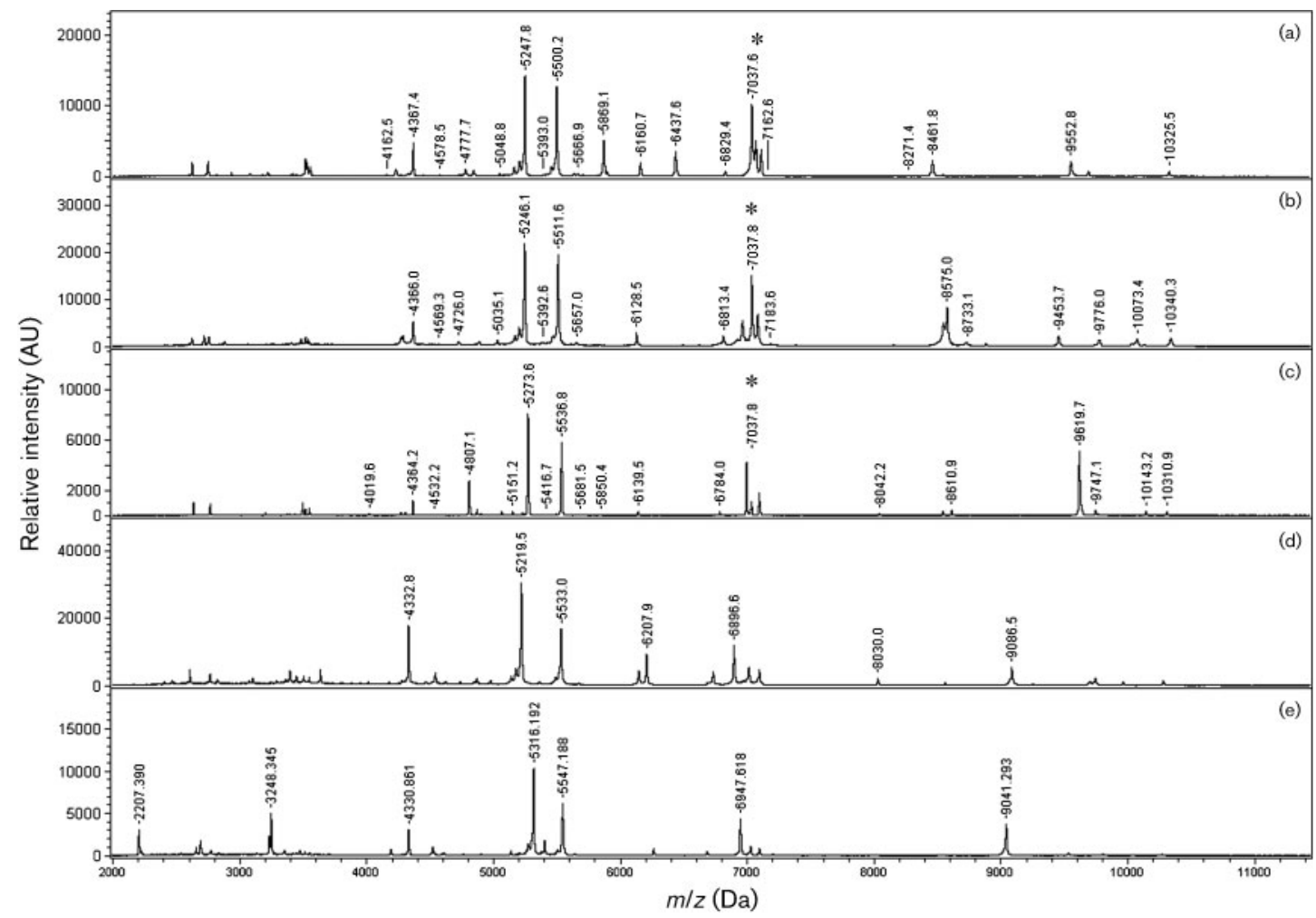

Fig. 3. MALDI-TOF MS profiles obtained from the analysis of C. jejuni (a), C. coli (b), C. lari (c), C. fetus (d) and C. hyointestinalis (e). The 50 S ribosomal L29 protein 'biomarker' (indicated by an asterisk). The relative intensities of the ions [in arbitrary units $(\mathrm{AU})]$ are shown on the $y$-axis, and the masses (in $\mathrm{Da}$ ) of the ions are shown on the $x$-axis. The $\mathrm{m} / z$ value stands for mass to charge ratio. For a single positive charge, this value corresponds to the molecular mass of the protein. 
lead to incorrect results (Kolinska et al., 2008). In another study, C. jejuni, C. coli and C. fetus were compared with $H$. pylori and $H$. mustelae by direct analysis of individual culture colonies in $50 \%$ methanol using MALDI-TOF MS. A few specific biomarker ions in the 10 to $20 \mathrm{kDa}$ range were reported to be the most discriminative of those observed (Winkler et al., 1999). However, in this study mass signal patterns were only observed in the 2 to $11 \mathrm{kDa}$ range for Arcobacter, Helicobacter and for Campylobacter.

For evaluation of the method, 144 clinical isolates were used in this study. In parallel to MALDI-TOF MS identification, all strains were analysed by PCR-RFLP. Identification results of the clinical isolates obtained by MALDI-TOF MS and PCR-RFLP are shown in Table 2. A list of $\log$ (score) data is available as Supplementary Table S1 with the online journal. All clinical isolates obtained from humans, the environment and from different farm animals, mostly poultry, gave sufficient spectra for species identification by MALDI-TOF MS. In total, MALDI-TOF MS identified all 144 clinical isolates at species level, i.e. $\log ($ score $) \geqslant 2.0$ and no differences in results were found associated with the source of the isolate. PCR-RFLP could not differentiate between C. fetus and C. hyointestinalis in three clinical isolates $(898,1147$ and 1307). In all these cases, a differentiation at the species level with MALDITOF MS was possible.

To investigate the stability of clinical isolate identification, 15 different strains were grown according to the standard procedure and subsequently stored at room temperature $\left(20{ }^{\circ} \mathrm{C}\right)$ and at $4{ }^{\circ} \mathrm{C}$, and tested after 2, 4, 6 and 9 days. In all cases, MS resulted in identical, correct identification results related to the reference database (data not shown). This finding supports the results of another investigation (Mellmann et al., 2008), where non-fermenting bacteria were stored at room temperature up to 7 days. This can be a great advantage if samples are collected together for a single run or where samples need to be reinvestigated. It is noteworthy that bacteria grown for $48 \mathrm{~h}$ on COS or CAM agar gave the best results, e.g. good quality spectra, reliable $\log$ (score). However, bacteria grown longer than $72 \mathrm{~h}$ gave poor spectra profiles, lower intensity and unspecific peaks. Nevertheless, in most cases correct identification of the bacteria could be obtained. In another study MALDITOF MS was used for identification of Listeria, it was reported that extended periods of growth (4 days tested) did not affect spectra quality and results (Barbuddhe et al., 2008). Obviously, this is species dependent. However, in a separate study E. coli was analysed using MALDITOF MS, where growth time did not affect the bacterial molecular profile significantly, hence, the incubation time span was up to $48 \mathrm{~h}$ (Mazzeo et al., 2006).

To determine the influence of cultivation media on the quality of spectra, the 15 clinical strains were grown on different types of solid media used to isolate such bacteria. Species identification was possible at the species level if bacteria were grown on CAM agar, which was not used to create the reference database. Interestingly Arcobacter, Helicobacter and Campylobacter grown on mCCD agar could not be used for MALDI-TOF MS species identification as very poor spectra patterns were obtained, if any; consequently, no identification was possible (data not shown). Apparently, mCCD agar contaminants interfere with ionization of biomolecules of the bacteria. In fact, in some cases it was not possible to pick up bacteria biomass from mCCD agar because the bacteria were strongly attached on the surface of this agar. The importance of mCCD agar could not be overlooked as a drawback of MALDI-TOF MS as this agar is commonly used to isolate strains of Campylobacteraceae. This means that additional subculturing is necessary if the bacteria are going to be used for MALDI-TOF MS identification. To the best of our knowledge, no study reporting results on the use of mCCD agar for micro-organism identification by MALDI-TOF MS has been published.

Altogether, these data show that MALDI-TOF MS fingerprinting is a fast and reliable method for the identification of Arcobacter and Helicobacter species, and their distinction from phenotypically similar Campylobacter species with applications in clinical diagnostics. As a result, considering the speed with which reliable identification can be obtained, this technique is well suited for large-scale research and diagnostic analyses.

\section{ACKNOWLEDGEMENTS}

The authors would like to thank Thomas Maier, Bruker Daltonik $\mathrm{GmbH}$, Bremen (Germany), for technical support on the MALDITOF MS and suggestions on the manuscript.

\section{REFERENCES}

Atabay, H. I., Corry, J. E. \& On, S. L. (1998). Identification of unusual Campylobacter-like isolates from poultry products as Helicobacter pullorum. J Appl Microbiol 84, 1017-1024.

Barbuddhe, S. B., Maier, T., Schwarz, G., Kostrzewa, M., Hof, H., Domann, E., Chakraborty, T. \& Hain, T. (2008). Rapid identification and typing of Listeria species by matrix-assisted laser desorption ionization-time of flight mass spectrometry. Appl Environ Microbiol 74, 5402-5407.

Bohr, U. R. M., Primus, A., Zagoura, A., Glasbrenner, B., Wex, T. \& Malfertheiner, P. (2002). A group-specific PCR assay for the detection of Helicobacteraceae in human gut. Helicobacter 7, 378-383.

Castera, L., Pedeboscq, A., Rocha, M., Le Bail, B., Asencio, C., de Lédinghen, V., Bernard, P. H., Laurent, C., Lafon, M. E. \& other authors (2006). Relationship between the severity of hepatitis C virus-related liver disease and the presence of Helicobacter species in the liver: a prospective study. World J Gastroenterol 12, 7278-7284.

Ceelen, L. M., Decostere, A., Chiers, K., Ducatelle, R., Maes, D. \& Haesebrouck, F. (2007). Pathogenesis of Helicobacter pullorum infections in broilers. Int J Food Microbiol 116, 207-213.

Claydon, M. A., Davey, S. N., Edwards-Jones, V. \& Gordon, D. B. (1996). The rapid identification of intact microorganisms using mass spectrometry. Nat Biotechnol 14, 1584-1586. 
EFSA (2005). Scientific Report of the Scientific Panel on Biological Hazards on the Request from the Commission Related to Campylobacter in Animals and Foodstuffs, annex to the EFSA Journal 2005, vol. 173, pp. 1-105. Parma: European Food Safety Authority.

Fagerquist, C. K., Bates, A. H., Heath, S., King, B. C., Garbus, B. R., Harden, L. A. \& Miller, W. G. (2006). Sub-speciating Campylobacter jejuni by proteomic analysis of its protein biomarkers and their posttranslational modifications. J Proteome Res 5, 2527-2538.

Jauk, V., Neubauer, C., Szolgyenyi, W. \& Vasicek, L. (2003). Phenotypic and genotypic differentiation of Campylobacter spp. isolated from Austrian broiler farms: a comparison. Avian Pathol 32, 33-37.

Kolinska, R., Drevinek, M., Jakubu, V. \& Zemlickova, H. (2008). Species identification of Campylobacter jejuni ssp. jejuni and C. coli by matrix-assisted laser desorption/ionization time-of-flight mass spectrometry and PCR. Folia Microbiol (Praha) 53, 403-409.

Kuijper, E. J., Stevens, S., Imamura, T., De Wever, B. \& Claas, E. C. J. (2003). Genotypic identification of erythromycin-resistant Campylobacter isolates as Helicobacter species and analysis of resistance mechanism. J Clin Microbiol 41, 3732-3736.

Mandrell, R. E., Harden, L. A., Bates, A., Miller, W. G., Haddon, W. F. \& Fagerquist, C. K. (2005). Speciation of Campylobacter coli, C. jejuni, C. helveticus, C. lari, C. sputorum, and C. upsaliensis by matrixassisted laser desorption ionization-time of flight mass spectrometry. Appl Environ Microbiol 71, 6292-6307.

Marshall, S. M., Melito, P. L., Woodward, D. L., Johnson, W. M., Rodgers, F. G. \& Mulvey, M. R. (1999). Rapid identification of Campylobacter, Arcobacter, and Helicobacter isolates by PCR-restriction fragment length polymorphism analysis of the 16S rRNA gene. J Clin Microbiol 37, 4158-4160.

Mazzeo, M. F., Sorrentino, A., Gaita, M., Cacace, G., Di Stasio, M., Facchiano, A., Comi, G., Malorni, A. \& Siciliano, R. A. (2006). Matrixassisted laser desorption ionization-time of flight mass spectrometry for the discrimination of food-borne microorganisms. Appl Environ Microbiol 72, 1180-1189.

Mellmann, A., Cloud, J., Maier, T., Keckevoet, U., Ramminger, I., Iwen, P., Dunn, J., Hall, G., Wilson, D. \& other authors (2008). Evaluation of matrix-assisted laser desorption ionization-time-offlight mass spectrometry in comparison to $16 \mathrm{~S}$ rRNA gene sequencing for species identification of nonfermenting bacteria. J Clin Microbiol 46, 1946-1954.
Neubauer, C. \& Hess, M. (2006a). Detection and identification of food-borne pathogens of the genera Campylobacter, Arcobacter and Helicobacter by multiplex PCR in poultry and poultry products. $J$ Vet Med B Infect Dis Vet Public Health 53, 376-381.

Neubauer, C. \& Hess, M. (2006b). Tissue tropism of Helicobacter pullorum in specific pathogen-free chickens determined by culture and nucleic acid detection. Avian Dis 50, 620-623.

On, S. L. W. (1996). Identification methods for Campylobacters, Helicobacters, and related organisms. Clin Microbiol Rev 9, 405-422.

Prouzet-Mauleon, V., Labadi, L., Bouges, N., Menard, A. \& Megraud, F. (2006). Arcobacter butzleri: underestimated enteropathogen. Emerg Infect Dis 12, 307-309.

Rocha, M., Avenaud, P., Menard, A., Le, B. B., Balabaud, C., BioulacSage, P., de Magalhaes Queiroz, D. M. \& Megraud, F. (2005). Association of Helicobacter species with hepatitis C cirrhosis with or without hepatocellular carcinoma. Gut 54, 396-401.

Stanley, J., Linton, D., Burnens, A. P., Dewhirst, F. E., On, S. L. W., Porter, A., Owen, R. J. \& Costas, M. (1994). Helicobacter pullorum sp. nov. - genotype and phenotype of a new species isolated from poultry and from human patients with gastroenteritis. Microbiology 140, 3441-3449.

Suh, M. J. \& Limbach, P. A. (2004). Investigation of methods suitable for the matrix-assisted laser desorption/ionization mass spectrometric analysis of proteins from ribonucleoprotein complexes. Eur J Mass Spectrom 10, 89-99.

Vandenberg, O., Dediste, A., Houf, K., Ibekwem, S., Souayah, H., Cadranel, S., Douat, N., Zissis, G., Butzler, J. P. \& Vandamme, P. (2004). Arcobacter species in humans. Emerg Infect Dis 10, 1863-1867.

Wilson, D. J., Gabriel, E., Leatherbarrow, A. J., Cheesbrough, J., Gee, S., Bolton, E., Fox, A., Fearnhead, P., Hart, C. A. \& Diggle, P. J. (2008). Tracing the source of campylobacteriosis. PLoS Genet 4, e1000203.

Winkler, M. A., Uher, J. \& Cepa, S. (1999). Direct analysis and identification of Helicobacter and Campylobacter species by MALDITOF mass spectrometry. Anal Chem 71, 3416-3419.

Young, V. B., Chien, C. C., Knox, K. A., Taylor, N. S., Schauer, D. B. \& Fox, J. G. (2000). Cytolethal distending toxin in avian and human isolates of Helicobacter pullorum. J Infect Dis 182, 620-623.

Zanoni, R. G., Rossi, M., Giacomucci, D., Sanguinetti, V. \& Manfreda, G. (2007). Occurrence and antibiotic susceptibility of Helicobacter pullorum from broiler chickens and commercial laying hens in Italy. Int $J$ Food Microbiol 116, 168-173. 